\title{
Late clinical outcome of suboptimal stent implantation defined by intracoronary optical coherence tomography
}

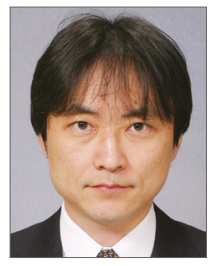

Takashi Kubo, MD, PhD; Takashi Akasaka*, MD, PhD

Department of Cardiovascular Medicine, Wakayama Medical University, Wakayama, Japan

Optical coherence tomography (OCT) is an intravascular imaging technique that provides high-resolution (10-20 $\mu \mathrm{m})$ images. During percutaneous coronary intervention (PCI), OCT offers useful information to guide treatment strategies and assess optimal stent implantation (Figure 1). However, OCT remains markedly underused in the real world. For further integration of OCT into clinical practice, it is necessary to accumulate evidence showing that OCT has a beneficial impact on the outcomes of PCI. In 2012 and 2015, Prati et $\mathrm{al}^{1,2}$ reported the clinical benefits of OCT guidance in PCI using data from a large retrospective registry named CLI-OPCI (Centro per la Lotta contro l'Infarto-Optimisation of Percutaneous Coronary Intervention). The CLI-OPCI study demonstrated that OCT guidance in PCI was associated with a significantly lower risk of cardiac death or myocardial infarction during a one-year follow-up period in comparison with angiography guidance alone ${ }^{1}$. Subsequently, the CLI-OPCI II study showed that OCT-defined suboptimal stent implantation, defined as in-stent minimum lumen area $<4.5 \mathrm{~mm}^{2}$, distal stent edge dissection $>200 \mu \mathrm{m}$, or significant reference vessel plaque and lumen area $<4.5 \mathrm{~mm}^{2}$ at either distal or proximal stent edges, was an independent predictor of device-oriented cardiovascular events (a composite of cardiac mortality, target vessel myocardial infarction, and target lesion revascularisation) during a one-year followup period ${ }^{2}$.

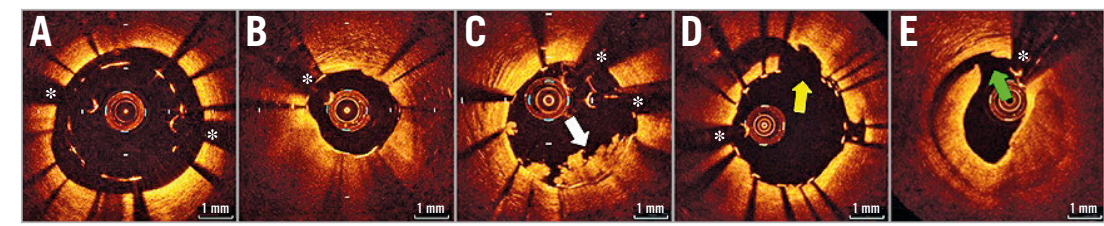

Figure 1. OCT images immediately after stent implantation. A) Stent malapposition. B) Stent underexpansion. C) In-stent tissue/thrombus protrusion (white arrow). D) Coronary artery dissection at stent edge (yellow arrow). E) Coronary artery dissection at reference segment (green arrow). * Guidewire artefact

*Corresponding author: Department of Cardiovascular Medicine, Wakayama Medical University, 811-1, Kimiidera, Wakayama, 641-8509, Japan.E-mail: akasat@wakayama-med.ac.jp 


\section{Present study}

In this issue of EuroIntervention, Prati et $\mathrm{al}^{3}$ report the results of the CLI-OPCI LATE study.

\section{Article, see page 443}

The CLI-OPCI LATE study evaluated the impact of OCTdefined suboptimal stent implantation at long-term follow-up. The authors demonstrated that: 1) during a median follow-up period of approximately three years, the presence of in-stent minimum lumen area $<4.5 \mathrm{~mm}^{2}$, distal stent edge dissection $>200 \mu \mathrm{m}$, or significant reference vessel plaque and lumen area $<4.5 \mathrm{~mm}^{2}$ at either distal or proximal stent edges was an independent predictor of device-oriented cardiovascular events; 2) conversely, in-stent minimum lumen area divided by mean reference lumen area $<70 \%$, acute stent malapposition, and intrastent plaque/thrombus protrusion were not associated with worse outcomes; and 3) the impact of the suboptimal OCT-defined stent implantation was confined to the early phases of follow-up, while the clinical outcome was comparable after the first year.

\section{OCT guidance in $\mathrm{PCI}$}

One major challenge for OCT is to develop criteria for optimal stent implantation. Previously, two pivotal studies used their own original OCT criteria for optimal stent implantation. The ILUMIEN III: OPTIMIZE PCI (OPtical Coherence Tomography Compared to Intravascular Ultrasound and Angiography to Guide Coronary stent Implantation: a Multicenter RandomiZEd Trial in Percutaneous Coronary Intervention) study defined acute procedural success as stent expansion of $\geq 90 \%$ (the minimum stent area of the proximal segment of the stent is $\geq 90 \%$ of the proximal reference lumen area, and the minimum stent area of the distal segment of the stent is $\geq 90 \%$ of the distal reference lumen area); no stent edge dissections classified as major (dissection with $\geq 60^{\circ}$ of the circumference of the vessel or $\geq 3 \mathrm{~mm}$ in length); and no stent malapposition categorised as major (incomplete stent apposition associated with unacceptable stent expansion $[<90 \%])^{4}$. The OPINION (OPtical frequency domain imaging versus INtravascular ultrasound in percutaneous coronary interventiON) study defined the goal of stent implantation as stent expansion of $\geq 90 \%$ (in-stent minimal lumen area is $\geq 90 \%$ of the average reference lumen area); complete apposition of the stent over its entire length against the vessel wall; symmetric stent expansion (minimum lumen diameter divided by maximum lumen diameter $\geq 0.7$ ); and no plaque protrusion, thrombus, and edge dissection with potential to provoke flow disturbances ${ }^{5}$. However, these OCT criteria were not determined based on the clinical evidence; therefore, their clinical impact is unclear. The CLI-OPCI LATE study identified significant OCT criteria for stent implantation associated with clinical outcomes. Further prospective study will be required to investigate whether OCT guidance using the criteria determined in the CLI-OPCI LATE study improves outcomes of PCI.

Lack of assessment of the clinical impact of OCT before PCI is a weakness of the CLI-OPCI LATE study. According to previous studies, pre-PCI lesion assessment by using OCT helps to predict complications during $\mathrm{PCI}^{6-8}$. OCT-derived lipid-rich plaque with a thin fibrous cap is potentially associated with the no-reflow/ slow-flow phenomenon after $\mathrm{PCI}^{6}$ and therefore may require the use of a distal protection device. A coronary bifurcation lesion with a small bifurcating angle and protruding carina has a high risk for side branch occlusion after main branch stent implantation $^{7}$ and therefore may need the use of a second wire for side branch protection. A heavily calcified lesion including a thick and circumferential calcification is likely to cause stent underexpan$\operatorname{sion}^{8}$ and therefore may warrant the use of a rotablator or cutting balloon. In addition, precise measurement of the reference lumen diameter and lesion length by using OCT helps to select the appropriate size of balloon and stent, which leads to a reduction in the incidence of tissue protrusion and stent edge dissection. Hence, OCT before PCI might play an important role for the guidance of $\mathrm{PCI}$ and induce changes in PCI procedures.

In the CLI-OPCI LATE study, $69 \%$ of the lesions with satisfactory angiographic results had OCT-defined optimal stent implantation. This suggests that the majority of lesions with satisfactory angiographic results do not require OCT assessment. OCT has some drawbacks including the cost of the imaging catheter and the additional time to perform imaging procedures. Therefore, routine use of OCT is not necessarily to be supported. Further efforts are needed to identify specific subgroups of patients in whom OCT is significantly effective for improving outcomes of PCI.

\section{Final remarks}

The CLI-OPCI LATE study determined the OCT criteria immediately after stent implantation for predicting late device-oriented cardiovascular events. The results of the CLI-OPCI LATE study will exert a direct influence on PCI optimisation strategies and also form a basis of future studies for OCT-guided PCI.

\section{Conflict of interest statement}

T. Kubo and T. Akasaka have received lecture fees and research funds from Abbott Vascular Japan Co., Ltd.

\section{References}

1. Prati F, Di Vito L, Biondi-Zoccai G, Occhipinti M, La Manna A, Tamburino C, Burzotta F, Trani C, Porto I, Ramazzotti V, Imola F, Manzoli A, Materia L, Cremonesi A, Albertucci M. Angiography alone versus angiography plus optical coherence tomography to guide decision-making during percutaneous coronary intervention: the Centro per la Lotta contro l'InfartoOptimisation of Percutaneous Coronary Intervention (CLI-OPCI) study. EuroIntervention. 2012;8:823-9.

2. Prati F, Romagnoli E, Burzotta F, Limbruno U, Gatto L, La Manna A, Versaci F, Marco V, Di Vito L, Imola F, Paoletti G, Trani C, Tamburino C, Tavazzi L, Mintz GS. Clinical Impact of OCT Findings During PCI: the CLI-OPCI II Study. JACC Cardiovasc Imaging. 2015;8:1297-305. 
3. Prati F, Romagnoli E, La Manna A, Burzotta F, Gatto L, Marco V, Fineschi M, Fabbiocchi F, Versaci F, Trani C, Tamburino C, Alfonso F, Mintz GS; on behalf of CLI-OPCI Project Investigators. Long-term consequences of optical coherence tomography findings during percutaneous coronary intervention: the Centro Per La Lotta Contro L'infarto - Optimization Of Percutaneous Coronary Intervention (CLI-OPCI) LATE study. EuroIntervention. 2018; 14:e443-51.

4. Ali ZA, Maehara A, Généreux P, Shlofmitz RA, Fabbiocchi F, Nazif TM, Guagliumi G, Meraj PM, Alfonso F, Samady H, Akasaka T, Carlson EB, Leesar MA, Matsumura M, Ozan MO, Mintz GS, Ben-Yehuda O, Stone GW; ILUMIEN III: OPTIMIZE PCI Investigators. Optical coherence tomography compared with intravascular ultrasound and with angiography to guide coronary stent implantation (ILUMIEN III: OPTIMIZE PCI): a randomised controlled trial. Lancet. 2016;388:2618-28.

5. Kubo T, Shinke T, Okamura T, Hibi K, Nakazawa G, Morino Y, Shite J, Fusazaki T, Otake H, Kozuma K, Ioji T, Kaneda H, Serikawa T, Kataoka T, Okada H, Akasaka T; OPINION Investigators. Optical frequency domain imaging vs. intravascular ultrasound in percutaneous coronary intervention (OPINION trial): one-year angiographic and clinical results. Eur Heart J. 2017;38:3139-47.

6. Tanaka A, Imanishi $\mathrm{T}$, Kitabata H, Kubo T, Takarada S, Tanimoto T, Kuroi A, Tsujioka H, Ikejima H, Komukai K, Kataiwa H, Okouchi K, Kashiwaghi M, Ishibashi K, Matsumoto H, Takemoto K, Nakamura N, Hirata K, Mizukoshi M, Akasaka T. Lipid-rich plaque and myocardial perfusion after successful stenting in patients with non-ST-segment elevation acute coronary syndrome: an optical coherence tomography study. Eur Heart J. 2009; 30:1348-55.

7. Watanabe M, Uemura S, Sugawara Y, Ueda T, Soeda T, Takeda Y, Kawata H, Kawakami R, Saito Y. Side branch complication after a single-stent crossover technique: prediction with frequency domain optical coherence tomography. Coron Artery Dis. 2014;25:321-9.

8. Kubo T, Shimamura K, Ino Y, Yamaguchi T, Matsuo Y, Shiono Y, Taruya A, Nishiguchi T, Shimokado A, Teraguchi I, Orii M, Yamano T, Tanimoto T, Kitabata H, Hirata K, Tanaka A, Akasaka T. Superficial Calcium Fracture After PCI as Assessed by OCT. JACC Cardiovasc Imaging. 2015;8:1228-9. 